\section{Introduction to Seismology}

\section{by Peter M Shearer}

Cambridge University Press, Cambridge June 2009; 410p., Price: \$64.99 (pbk)

ISBN: 978-05-21708-42-5

In the modern era of internet connectivity, there is a tendency among students to rely more and more on websites for locating information on the subjects of their immediate interest. However, I have always believed that there is no substitute for good textbooks that begin with basic principles like physical laws and background mathematical formulations and then trace the evolution of the subject to

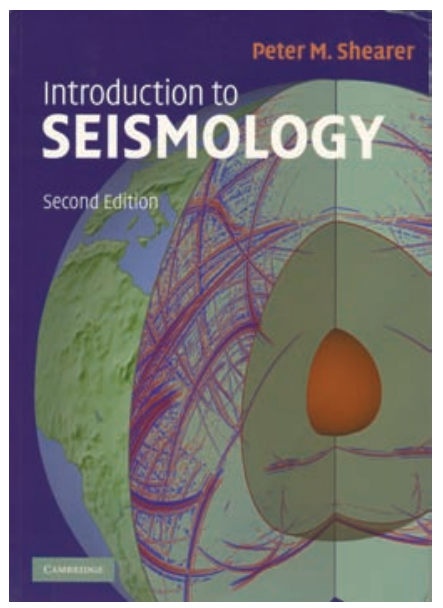

various applications. I am not a professional seismologist but my research endeavour to link the nature of resistivity distribution, by virtue of sensitivity of resistivity, to fluid content, can serve as useful proxy in tracking the fluid-induced rheological transitions and can thus explain the space-depth partitioning and focusing of hypocenters on sharp resistivity interfaces (brittle-ductile transitions). This resistivity-seismicity linkage has increased my need to understand the complex processes of seismogenesis. Therefore, my attempt to review the book Introduction to Seismology may be seen as an overview from a practitioner seismologist rather than as a specialist professional.

The book begins with a lucid and concise historical perspective of the young science of seismology that has been studied quantitatively. On the theoretical front, it primarily deals with the analysis of the propagation of elastic waves produced by earthquakes. The early distinction between body waves that travel through solid volumes and surface waves which travel along free surfaces, the approximations of the mode of seismic sources from explosive point to double couple source, the continued improvements in instrumentation from undamped pendulums to modern seismographs working on the principle of electromagnetic induction, the availability of data from the Worldwide Standardized Seismograph Network (WWSSN), and the rapid progress in determining seismic wave velocities has not only improved the mapping of earthquakes but also provided refined models of Earth's seismic velocity structure, leading to the present travel-time tables. The evidence of the Earth's liquid outer core, the concept of the shadow zone and seismic wave discontinuities associated with phase changes in crystal structures have been well recognized. The realization that earthquakes are not randomly distributed but tend to be concentrated along well defined belts helped toward the establishment of the theory of plate tectonics, which today provides a general framework for understanding the dynamics, focal mechanism, depth distribution of earthquakes, etc. This coupled with the advent of high-speed computers, more precise data, and knowledge obtained from the study of nuclear atomic explosions, has made possible an unparalleled growth in the field of seismology, allowing an ever increasing understanding of the earthquake generating processes and opening new gateways to imaging the Earth's interior from a global scale to the local level, permitting new insights into deep dynamics as well as the location of resources.

Following this background of Chapter 1 on seismology, the concept and computations of stress and strain are introduced in Chapter 2 as a measure of the internal forces and deformation that are central to understanding the physics of earthquakes and provide a quantitative base for describing seismic wave propagation in elastic solids. The equations denoting linear relationship between stress and strain lead to the definition of seismic $\mathrm{P}$ and $S$ wave velocities in terms of the intrinsic properties of the medium such as rigidity, density, Poisson ratio, etc. Chapter 3 then constructs and solves the formulae of seismic wave propagation in uniform space assuming perfect elasticity with no energy loss from attenuation. The solutions of plane, polarized and spherical waves are treated separately to provide expressions for compression and shear-wave velocities.
Ray theory continues to be extensively used to interpret seismic data, both for earthquake location and to characterize internal structures, and three full chapters are dedicated to the different facets of ray theory. In Chapter 4, starting from the extension of Snell's law of refraction in optics to seismic waves, the travel-time calculations for known velocity structures incorporating forward 1D, 3-D ray tracing are presented. The ray nomenclature for different seismic phases associated with the numerous possible ray geometries resulting from reflection and, or, refraction are then detailed with the help of stack plots of travel-times. The inversion of travel-times to obtain region specific optimum 1 -D velocity structures, and generalization to 3-D models using tomography are discussed in Chapter 5, incorporating linear and regularization approaches. The limitation of error distribution in inverted velocity determination resulting from the tacit assumption that source locations are known precisely is then addressed by the implementation of joint hypocenter determinations and the double-difference location algorithm. Chapter 6 deals with examination of the amplitude and phase of seismic waves to reveal additional information about the Earth's structure and seismic sources. Special focus is given to the use of ray theory to account for geometrical spreading effects, reflection and transmission coefficients at interfaces, and intrinsic attenuation. The physics and computation of the seismic hazard sensitive attenuation parameter $Q$ is elegantly introduced at this stage.

Having introduced the theoretical background, the rest of the book focus on applications of seismology for imaging the structures of the Earth as well as for earthquake source quantification. Chapter 7 deals with reflection seismology designed for imaging shallow depths for hydrocarbon or mineral exploration as well as the deep structures that constrain compositional and geodynamical processes. The principles of commonly adopted processing techniques, e.g. midpoint stacking, deconvolution, and migrations are discussed as a tool to enhance signal/noise ratios for shallow imaging, whereas the basics of receiver functions are outlined for the deep imaging of structures. The Kirchhoff theory is explained as an important technique for computing synthetic seismograms. Deviating from the use of body waves, the significance of the dispersion characteristic of surface waves are highlighted in Chapter 8, especially for Earth's shallow 
structure and low-frequency source properties.

Given some knowledge of source characteristic is essential for deducing seismic structures as well as gaining insights into the way in which radiated seismic energy is related to the physical properties of the source, Chapter 9 again deals with special techniques in resolving properties of the source. The concepts of Green's function and moment tensor are introduced to define radiation pattern, the nature of fault movement, the determination of centroid locations, the relation to 'beachball representation', directivity, stress drop, earthquake magnitude, etc. Finally, the steps of slip modelling and certain unresolved issues like linkage to heat flow anomalies, the location of earthquakes to relatively low shear-stress zones are pointed out. Chapter 10 deals with the still unfulfiled dreams of earthquake prediction. Some important leads in observing surface deformation using GPS, the relation of recurrence intervals to threshold stress levels, decay rates of aftershocks, 'b values', the search for earthquake precursors, and the hypothesis of dilatancy-diffusion to explain some precursors are briefly discussed but earthquakes remain difficult to predict. The concluding Chapter 11 summarizes some emerging trends in seismology, both in terms of advances in instrumentation, the characterization of frequency dependent noise level, anisotropy, shear wave splitting, etc.

The most outstanding aspect of the book is the organized structuring in each chapter, stating the governing physical laws. The strengths and limitations of the specific techniques used are highlighted. Often the directions of future research are outlined. I compliment the author for his excellent organization and maintainance of a balance between the mathematical details and the physical principles. Thoughtfully designed appendices and numerical exercises at the end of each chapter will be of added value to students and practitioners. The presentation flows well and is easy to follow. Although the book is designed to be covered in a singlesemester class, it may be difficult to do full justice to all its rich contents within that time constraint and it may be necessary to omit the flagged sections. Perhaps guided by personal interests and background, I should have liked further elaboration of the design of observational networks for the better estimation of earthquake locations for constraining regional seismotectonic models. Similarly, inclusion of some discussion on non-seismological precursory research as well as the role of geophysical imaging techniques in the deeper understanding of seismogenesis would have been useful in projecting seismology as a multi-disciplinary subject.

\section{B. $R$. Arora}

Wadia Institute of Himalayan Geology, 33 GMS Road, Dehradun 248 001, India

Email:arorabr@wihg.res.in

\section{Episodes is your window to the world. Subscribe today!}

\section{Episodes}

Name

(please print)

Address

$\begin{array}{ll}\text { City } & \text { State/Province } \\ \text { Country } \_ \text {Zip/Postal Code }\end{array}$

Please begin my subscription from:

$\begin{array}{rrrr}\text { March } & \text { June } & \text { Sept. } & \text { Dec. } \\ \text { Year } & \text { Year } & \text { Year } \quad \text { Year }\end{array}$

To start your subscription, fill in this form and mail to:

Episodes

Geological Society of India

No.63, 12th Cross, Basappa Layout

Gavipuram P.O., P.O. Box No. 1922

Bangalore - 560 019, INDIA

Telefax: +91-080-26613352; Phone:+91-080-22422943

Email: episodes.journal@gmail.com
Payment may be made by:

- Checks/Bank draft (US \$ only) payable to: 'Geological Society of India A/C Episodes'

- Bank Transfer:

'Geological Society of India A/C Episodes'

Bank Name : Canara Bank, Hanumanthanagar Branch Bangalore - 560019

S.B. A/C No. : 0472101044517

Bank Code : 560015020

Swift Code : CNRBINBBLFD

Annual Subscription rate: US \$30 